\title{
Intraoperative electroencephalographic burst suppression may help to indentify patients at risk for long-term adverse outcome: Findings from a homozygous twins case
}

\section{Federico Linassi}

Universita degli Studi di Padova Dipartimento di Medicina

Eleonora Maran ( $\square$ maraneleonora@gmail.com )

Universita degli Studi di Padova Dipartimento di Medicina https://orcid.org/0000-0003-4968-8611

\section{Matthias Kreuzer}

Klinikum rechts der Isar der Technischen Universitat Munchen Klinik fur Anasthesiologie und Intensivmedizin

\section{Anna Paola Mazzarolo}

Azienda ULSS n 2 Marca Trevigiana

\section{Edoardo Rosellini}

Università degli studi di Padova

\section{Paolo Zanatta}

Universita degli Studi di Verona

\section{Michele Carron}

Universita degli Studi di Padova Dipartimento di Medicina

\section{Case report}

Keywords: Intraoperative monitoring; Consciousness monitors; Postoperative cognitive dysfunction.

Posted Date: August 13th, 2019

DOI: https://doi.org/10.21203/rs.2.417/v2

License: (9) This work is licensed under a Creative Commons Attribution 4.0 International License. Read Full License

Version of Record: A version of this preprint was published at Anaesthesia Critical Care \& Pain Medicine on October 1st, 2020. See the published version at https://doi.org/10.1016/j.accpm.2019.12.012. 


\section{Abstract}

Background: Postoperative cognitive dysfunction (POCD) presents a risk factor after general anesthesia, especially for old or cognitively impaired patients. Hence, strategies to identify possible patients at risk could help to decrease the rate of POCD. In this case report we relate the occurrence of electroencephalographic burst suppression and low bispectral indices to the development of neurocognitive decline after 12 months in one homozygous twin, whereas the other twin exhibited neither burst suppression nor neurocognitive decline. Case presentation: Two 68-year-old homozygous twins underwent the same surgical procedure with the same anesthetic regimens in comparable concentrations. For cognitive assessment, we applied a battery of neurocognitive tests at baseline (one day before surgery), at hospital discharge (three days after surgery), and 12 months after the procedure. The twins performed similarly in preoperative and discharge testing, but one twin showed worse test performance after 12 months. During surgery, we tested for possible connected consciousness by applying the isolated forearm technique (IFT). For assessment of the level of anesthesia and visual inspection of the EEG, we used the bispectral index (BIS). Neither of the twins showed signs of connected consciousness during anesthesia and the emergence tone to the return of a positive IFT response were similar. We observed lower intraoperative BIS indices for one twin. Furthermore, this twin also had two episodes of Burst Supppression. Vital parameters, particularly mean blood pressure, were stable and comparable during the entire duration of anaesthesia. The twin who exhibited Burst Suppression and lower BIS indices showed distinctly worse cognitive test scores after 12 months compared to baseline and discharge testing, indicating serious neurocognitive decline. The other twin's cognitive performance did not decrease. Conclusions: We present findings from a unique scenario of homozygous twins with comparable lifestyles undergoing the same surgical intervention. The twin with neurocognitive decline had lower BIS values and multiple episodes of BSupp. These findings suggest that EEG monitoring under general anesthesia could help to identify frail brains that may be at increased risk of developing cognitive impairments.

\section{Background}

Over the next years, the number of senior patients and patients with cognitive impairments will increase drastically. As a consequence, the number of patients with neurocognitive impairments after anesthesia will increase as well. ${ }^{1}$ In order to develop strategies that can help to minimize these adverse outcomes, intraoperative electroencephalographic (EEG) monitoring could prove helpful. ${ }^{2}$ The prevention of very low EEG-based monitoring indices, such as the bispectral index (BIS, Covidien, Boulder CO) and burst suppression (BSupp) patterns, represents an especially promising approach. In fact, deep hypnotic levels represented by low indices and/or BSupp have been independently associated with postoperative mortality, ${ }^{3-5}$ but their influence on postoperative cognitive dysfunction (POCD) and delirium (POD) has not been established. ${ }^{6-7}$ Episodes of BSupp have also been associated with delirium in the postoperative area unit ${ }^{8}$ and POD. ${ }^{9-10}$ In general, the relationship between deep anesthesia (with BSupp) and outcome is still a matter of controversial discussion. 
In addition to the consequences of excessively deep sedation, anesthesia of inadequate depth could trigger intraoperative awareness events ${ }^{11}$ that could also lead to long-tern neurocognitive decline because of post-traumatic consequences. ${ }^{12}$

Here we present a case of senior homozygous twins with similar lifestyle that underwent the same surgical intervention, with one twin developing postoperative cognitive dysfunction. We evaluated intraoperative BIS values, visually-identified BSupp episodes, vital parameters, and drug concentrations to find a possible anaesthesia-related explanation for the differences in outcome between the brothers.

\section{Case Presentation}

We present the case of two Italian 68-year-old male homozygous twins (S1 and S2) who underwent general anaesthesia for total thyroidectomy. The twins have the same level of education (5 years in school), and both worked as hodmen and lived on different floors in the same house with their families. Their weight and height were similar as well: $73 \mathrm{~kg}$ and $174 \mathrm{~cm}$ (S1) and $76 \mathrm{~kg}$ and $175 \mathrm{~cm}$ (S2). Both twins had essential hypertension that was well controlled with beta-blockers. Prior to this intervention, neither of the brothers had undergone surgery or received general anesthesia.

\section{Procedure}

For thyroidectomy, the procedures for each twin were similar. Neither received premedication, and anesthesia was induced with fentanyl $150 \mathrm{mcg}$ and propofol $2 \mathrm{mg} \mathrm{kg}^{-1}$. For neuromuscular blockade (NMB), we delivered $1 \mathrm{mg} \mathrm{kg}^{-1}$ of rocuronium. After orotracheal intubation, we maintained anesthesia with desflurane at a minimum alveolar concentration (MAC) of 0.8 (5.4\% vaporizer setting on the Dräger Zeus ${ }^{\circledR}$ Infinity ${ }^{\circledR}$ Empowered anaesthesia machine). We further administered two fentanyl boluses: 150 mcg just before skin incision and $100 \mathrm{mcg} 40$ minutes later. We also gave rocuronium boluses of 0.15 mg kg-1 to maintain a deep NMB, defined as absence of first twitch at train-of-four stimulation. Thirty minutes before the end of surgery, the patients received paracetamol $1000 \mathrm{mg}$, ketoprofen $160 \mathrm{mg}$, and ondansetron $4 \mathrm{mg}$ for pain, nausea and vomiting prophylaxis, respectively. At the end of surgery, we reversed the deep NMB with sugammadex $\left(4 \mathrm{mg} \mathrm{kg}^{-1}\right)$ and discontinued desflurane delivery.

\section{Monitoring}

We monitored level of anesthesia in both twins with a BIS monitor by applying a BIS Quatro Sensor to the forehead. The display of the raw EEG on the BIS monitor also allowed for visual inspection for intraoperative BSupp episodes. We used the isolated forearm technique (IFT) to test for possible episodes of connected consciousness and the train-of-four (TOF) watch acceleromyography SX (Organon, Ireland) monitoring throughout anaesthesia to assess the state of NMB. We attached BIS and TOF monitors 
before anaesthesia induction according to the manufacturer specification and removed them after anaesthesia emergence. We placed the tourniquet for IFT monitoring on the dominant (right) arm before induction. It was inflated after injection of propofol and fentanyl, but before the patients received rocuronium. We deflated the tourniquet only after complete reversal of NMB (verified by TOF) at the end of surgery. We performed IFT testing at 5-minute intervals throughout surgery. To facilitate this, each patient wore headphones through which the following prerecorded voice message was played: "Name, name, it's your anaesthesiologist speaking. If you can hear me, squeeze your hand... [10 seconds pause] ... Name, name, it's your anaesthesiologist speaking. If you feel pain, squeeze your hand twice" (with "name" replaced by the name of the patient).

\section{Cognitive assessment}

Both twins underwent the same neuropsychological tests at three different times: the day before surgery (T0), 3 days after surgery (T1), and 12 months after surgery (follow-up). We performed (i) Montreal Cognitive Assessment (MoCA), (ii) Trail Making Test A (TMT-A) and B (TMT-B), (iii) Forward and Backward Digit Span Tests (DST), and (iv) the Frontal Assessment Battery (FAB). These tests are designed to assess cognitive function, including short-term memory, working memory, visuo-spatial research ability, selective and divided attention, psychomotor speed and executive functions. After correcting the scores for age and level of schooling, a psychologist (APM) interpreted the results. We considered both cut-off values (26/30 for MoCA, 94 seconds for TMT-A, 283 seconds for TMT-B, 3.75/9 for DST Forward, and 3/9 for DST Backward) as well as z-scores (for FAB) for the evaluation of cognitive status. Cognitive deficit was defined as a score below the cut-off value for the MoCA test and DST, a score above the cut-off for the TMT tests, or a z-score of at least -1.0 for the FAB test (i.e., a reduction of one or more standard deviations in FAB performance). We further assessed the level of sedation during anesthesia emergence with the Richmond Agitation-Sedation Scale-RASS. ${ }^{13}$

\section{Results}

Neither twin required vasoconstrictor drugs during general anaesthesia, as their intraoperative haemodynamic parameters were stable. Neither twin reported explicit recall. The duration of surgery was very similar (77 minutes for $\mathrm{S} 1$ and 84 for $\mathrm{S} 2$ ).

\section{Monitoring results}

Before induction of anaesthesia, the BIS values for S1 and S2 were 97 and 98, respectively. The mean BIS values during maintenance phase were 35 for $\mathrm{S} 1$ and 42 for $\mathrm{S} 2$. Furthermore, we visually identified two episodes of BSupp in patient S1: the first episode occurred after skin incision and lasted 12 minutes, and 
the second occurred approximately 40 minutes after skin incision (after the $100 \mathrm{mcg}$ fentanyl bolus) and lasted 8 minutes. No BSupp was observed for S2. Intraoperative BIS values are summarized in Table 1.

Neither patient exhibited a positive IFT response during surgery. S1 had a positive response 8 minutes after discontinuing desflurane and was extubated 3 minutes later. He was confused (Richmond AgitationSedation Scale-RASS: -2) for approximately 10 minutes after awakening. S2 had a positive IFT response 6 minutes after discontinuing desflurane and was extubated 4 minutes later. He was confused (RASS: -3) for approximately 5 minutes after awakening. Neither patient exhibited arm complications related to ischemia induced by the tourniquet.

Intraoperative monitoring values during surgery are shown in Table 1.

\section{Neurocognitive testing results}

Preoperatively, scores obtained at MoCA, FAB tests and Backward DST fulfilled the definition of (mild) cognitive deficit for both twins. 3 days after the operation, all test scores were worse compared to the preop tests in both twins, except for the Forward and Backward DST scores which remained the same. The scores were similar between the brothers for these assessments. At the 12-month follow-up, the neurocognitive test results for $\mathrm{S} 2$ were similar to his postoperative results. S1, in contrast performed worse in all tests. All scores fulfilled (or almost fulfilled [TMT-A score was at the cut-off value]) the definition of neuro-cognitive dysfunction.

Neurocognitive test results are shown in Table 1.

\section{Table 1: Neurocognitive test results and intraoperative monitoring values.}

BIS: bispectral index; DST: Digit Span Test; FAB: Frontal Assessment Battery; IFT: isolated forearm technique; MoCA: Montreal Cognitive Assessment; TMT: Trail Making Test. Neurocognitive results in parentheses $(\mathrm{c})$ represent age- and schooling-corrected values. 


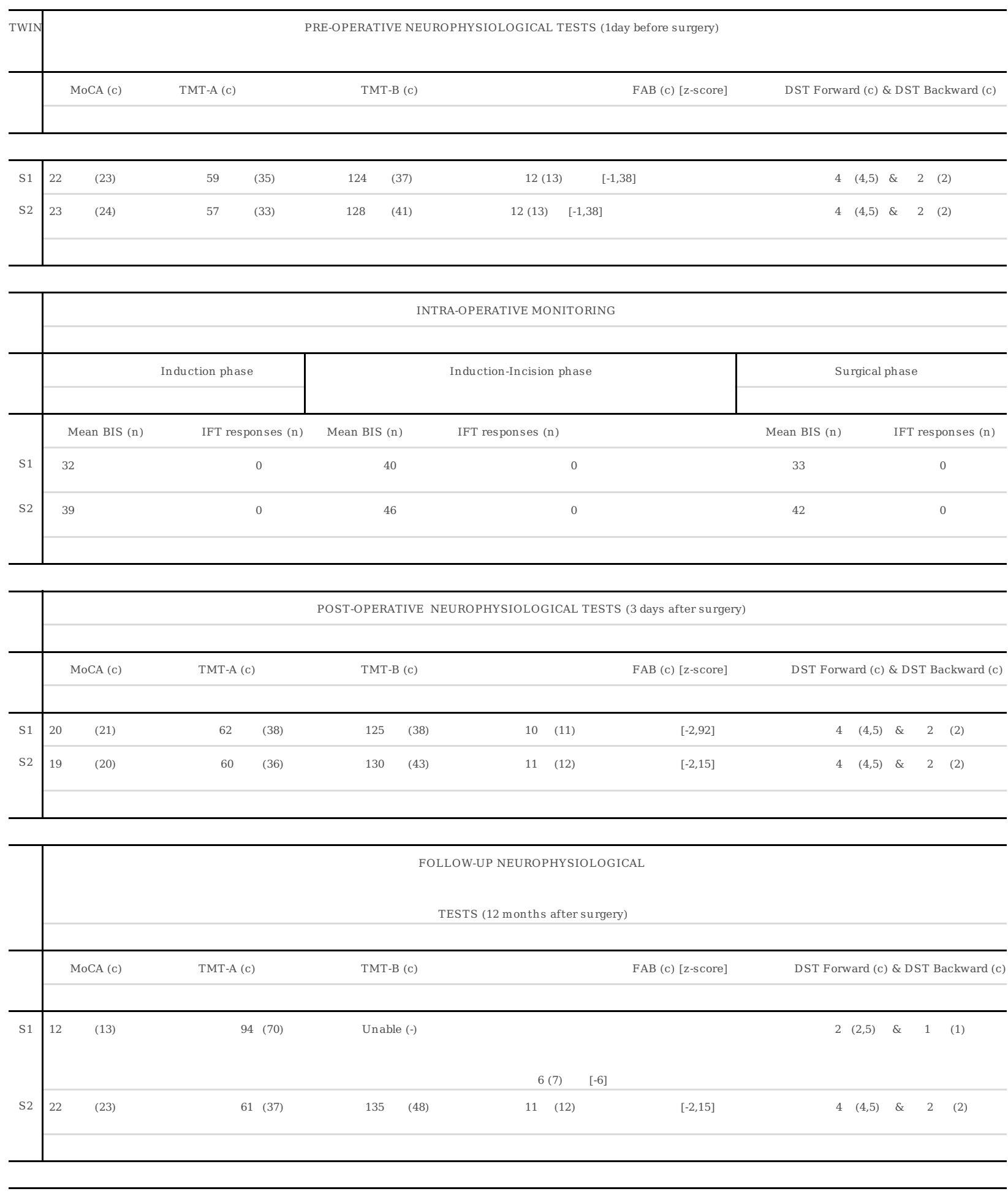

\section{Discussion And Conclusions}

In this case report we describe two patients, homozygous twins, who had the same medical history, and received the same anaesthesia regimen for the same surgical intervention (total thyroidectomy). The intervention duration was similar, as were the vital parameters (particularly the lack of hypertension). The brothers did not have excessively light levels of general anaesthesia, i.e., no connected consciousness as tested with IFT. They also had similar perioperative and postoperative (3 days) neurocognitive test results. Nevertheless, S1 performed substantially worse in neurocognitive tests one year after surgery. 
Short-term memory, working memory, and frontal functions (conceptualization, mental flexibility, motor programming, sensitivity to interference, inhibitory control, environmental autonomy) were most affected.

We observed lower BIS values and episodes of BSupp in S1, but not in S2. Since low BIS and BSupp seem associated with a higher risk of adverse outcomes, ${ }^{3-5,8-10}$ our finding may present an unmasking of a frail brain.

The cognitive decline of S1 after 12 months was not predicted by any of the peri-operative tests. Both twins performed in a similar fashion before surgery and in the 3-day postop test.

In our opinion, given that these two homozygous twins underwent the same surgical procedure and anaesthesia protocol, the fact that only the one with BSupp had a worse 12-months outcome suggests that his brain was more susceptible to anaesthetic drugs than the other's. This could mean that the generation of BSupp may have unmasked the more fragile brain of S1, even if there was no development of cognitive impairment at the first days following general anaesthesia.

Besides the unique opportunity to observe the postoperative cognitive function, the brothers presented a risk patient group for an adverse cognitive outcome. They were of older age ( $>65$ years), received limited education, and showed signs of mild cognitive impairment before surgery. ${ }^{1}$ Despite this, only one brother - the one with BSupp - developed POCD. Hence, our result may also point towards the necessity of longer follow-up periods for patients at risk. Also, BSupp did not occur in situations of hypotension that would have made our case a triple low case ${ }^{14}$. In the current state of the literature where the role of Bsupp and neurocognitive outcome is still debated, ${ }^{6-7}$ our case report suggests a possible association between low BIS/BSupp and long-lasting neurocognitive consequences.

Of course, our observation is just a case report and is significantly limited by the lack of additional neuropsychological follow-up evaluations in the postoperative period closer to surgery as well as later on. However, 18 months after surgery, S2 has continued living at home while S1 had to be transferred to a long-term-stay hospital for elderly. Furthermore, our BIS and EEG data were only observational and were assessed by visual inspection. Hence, we cannot compare the data of these patients to those of other subjects.

In conclusion, we would like to state that our observation shows the potential of intraoperative BSupp identification in helping to unmask a frail brain at risk for (long-term) cognitive impairments.

\section{List Of Abbreviations}

IFT: Isolated forearm technique

BIS: Bispectral Index

BSupp: Burst Suppression 
POD: Postoperative delirium

POCD: Postoperative cognitive dysfunction

MAP: Mean arterial pressure

NMB: neuromuscular blockade

TOF: train-of-four

MoCA: Montreal Cognitive Assessment

TMT: Trail Making Test

DST: Digit Span Test

FAB: Frontal Assessment Battery

RASS: Richmond Agitation-Sedation Scale

\section{Declarations}

\section{Ethical approval and consent to participate}

Both patients (S1 and S2) provided written informed consent for participation in this case report. Ethical approval was not applicable since the monitoring and treatments of patients involved did not differ from routine clinical practice at our hospital.

\section{Consent to publish}

Written informed consent was obtained from the participants for publication of this article and any accompanying tables/images.

\section{Availability of data and materials}

This case report contains clinical data from medical records in our hospital. All the relevant data for this case-report, reported anonymously, have been inserted in the Table 1.

The datasets used in this current case-report, as well as the signed consent to participate and consent to publish, are available from the corresponding author upon reasonable request.

\section{Competing interests}

There are no competing nor financial interests.

\section{Funding}


No financial support was received for this work.

\section{Author's contributions:}

FL and PZ conceived of the study; acquired, collected, and analysed data; and drafted and revised the final manuscript. APM, EM and ER collected and analysed data, and revised the final manuscript. MK participated in analysing the data, discussing the results, and revising the final manuscript. MC participated in conceiving the study, analysing the data, discussing the results, and revising the final manuscript. All authors read and approved the manuscript.

\section{Acknowledgements}

The authors would like to thank Mila Dorji, of the Yale University, for his contribution in revising the final manuscrpit.

\section{References}

1. Needham MJ, Webb CE, Bryden DC. Postoperative cognitive dysfunction and dementia: what we need to know and ro. BJA 2017; 119: i115-i125. 2. Chan MT, Cheng BC, Lee TM, Gin T; CODA Trial Group. BISguided Anesthesia Decreases Postoperative Delirium and Cognitive Decline. J Neurosurg Anesthesiol 2013; 25: 33-42 3. Monk TG, Saini V, Weldon BC, Sigl JC. Anesthetic management and one-year mortality after noncardiac surgery. Anesth Analg 2005; 100:4-10 4. Kertai MD, Pal N, Palanca BJ et al. Association of perioperative risk factors and cumulative duration of low bispectral index with intermediate-term mortality after cardiac surgery in the B-Unaware Trial. Anesthesiology 2010; 112: 1116-27 5. Leslie K, Myles PS, Forbes A, Chan MT. The effect of bispectral index monitoring on long-term survival in the Baware trial. Anesth Analg 2010; 110:816-22 6. Steinmetz J, Funder KS, Dahl BT, Rasmussen LS. Depth of anaesthesia and post-operative cognitive dysfunction. Acta Anaesthesiol Scand 2010; 54:162-8 7. Hou R, Wang H, Chen L, Qiu Y, Li S. POCD in patients receiving total knee replacement under deep vs light anesthesia: A randomized controlled trial. Brain Behav 2018; 30: e00910 8. Hesse S, Kreuzer M, Hight D, Gaskell A, Devari P, Singh D, Taylor NB, Whalin MK, Lee S, Sleigh JW, Garcia PS. Association of electroencephalogram trajectories during emergence from anesthesia with delirium in the postanesthesia care unit: an early sign of postoperative complications. BJA, 2019; 122:622-634 9. Fritz BA, Kalarickal PL, Maybrier HR, Muench MR, Dearth D, Chen Y, Escalier KE, Ben Abdallah A, Lin N, Avidian MS. Intraoperative electroencephalogram suppression predicts postoperative delirium. Anesth Analg 2016 122: 234-42 10. Soehle M, Dittmann A, Ellerkmann RK, Baumgarten G, Putensen C, Guenther U. Intraoperative burst suppression is associated with postoperative delirium following cardiac surgery: a prospective, observational study. BMC anesthesiol 2015; 28: 15-61 11. Linassi F, Zanatta P, Tellaroli P, Ori C, Carron M. Isolated forearm technique: a meta-analysis of connected consciousness during different general anaesthesia regimens. Br J Anesth 2018; 121: 198-209. 12. Osterman JE, Hopper J, Heran WJ, Keane TM, van der Kolk BA. Awareness under anesthesia and the development of posttraumatic stress disorder. Gen Hosp Psychiatry 2001; 23: 198-204 13. N. Sessler CN, Gosnell MS, Jo Grap M, et al. The Richmond Agitation-Sedation Scale. American Journal of Respiratory and Critical Care Medicine 2002; 166:10 14. 
Sessler DI, Sigl JC, Kelley SD, et al. Hospital stay and mortality are increased in patients having a "triple low" of low blood pressure, low bispectral index, and low minimum alveolar concentration of volatile anesthesia. Anesthesiology 2012; 116: 1195-203.

\section{Supplementary Files}

This is a list of supplementary files associated with this preprint. Click to download.

- supplement1.pdf

- supplement2.jpg 